operations. Firstly, the incision must be through skin not far distant from the anus, and intestinal bacteria are more widely distributed over this area than is commonly realized; among them the spores of clostridia are resistant to ordinary methods of skin disinfection. Secondly, the operation entails severing large masses of muscle in which bacteria from the region of the incision can be directly implanted. Thirdly, and most important, the disease which has necessitated the operation reduces the blood supply to the area, and may thus reduce the oxygen tension to a level permitting clostridial growth. The degree to which this area is so affected is doubtless one variable factor determining the outcome. Others are the efficacy of skin cleansing and the toxigenicity of the patient's own strain of $\mathrm{Cl}$. welchii. This is a property in which different strains vary widely.

This operation carries the imperative indication for penicillin cover in doses allowing for the fact that clostridia are less sensitive to penicillin than, for instance, streptococci. At least 500,000 units should be given at intervals of not more than six hours for seven days." Other operations with a similar risk meriting penicillin cover were recently referred to in our correspondence columns by $\mathrm{M}$. J. Parker. ${ }^{5}$

Gas gangrene is a rare and terrifying condition, and it is perhaps natural that operating-theatre staffs should regard it as calling for exceptional measures. In fact it is much easier to disinfect a theatre after a case of gas gangrene than after a dirty operation on the lower bowel, because in the former the organisms are all in the vulnerable vegetative form, whereas in faeces they exist largely as resistant spores. That most strains from the normal bowel are as toxigenic as those which have had the opportunity of causing gas gangrene has been shown by D. J. E. Price and R. A. Shooter. ${ }^{6}$ It remains to be seen whether conditions in the operating-theatres concerned in these recent episodes are considered to require improvement. What can be said with some confidence is that when gas gangrene follows thigh amputation there is no need to look any further for the source of infection than the patient's anus.

\section{Standardization in Human Cytogenetics}

The explosion in our knowledge of human chromosomes and their abnormalities which has occurred during the past ten years has resulted in many difficulties, particularly of communication and nomenclature. There have now been three conferences to consider these problems. The first of these was in Denver in 1960, the second in London in 1963, while the third and last was convened at the University of Chicago in September 1966. The aim of each of these meetings has been to improve communication and understanding in the field of human cytogenetics. At Denver order was brought into the system of numbering human chromosomes, and the international system agreed at that meeting has remained essentially unaltered since. In London the Denver system was consolidated and the alternative use of letters to classify the different chromosome groups, which had been advocated by some workers since 1961, was agreed internationally to be a useful alternative to the Denver system of nomenclature. At the conference it became clear that little progress had been

- Chicago Conference: Standardization in Human Cytogenetics. Birth Defects, Original Article Series, 1966, Vol. 2, No. 2, New York. Copies can be obtained free from the Medical Department, The National Foundation-March of Dimes, 800 Second Avenue, New York, N.Y. 10017 . made in chromosome identification over the three preceding years.

The report of the Chicago Conference, published recently, is perhaps one of the most important documents published in the field of human cytogenetics. It considers new means for describing the normal chromosomes and deviations from the normal complement, and proposes an entirely new system of notation for the uniform description of the human chromosome set and its abnormalities. This shorthand system is also applicable to the description of the somatic chromosomes of non-human species, so that perhaps from this conference a universal system of notation may eventually develop. The system is most carefully designed so that it should be adaptable to automated data processing and information retrieval systems, which are becoming more and more widely used.

Other topics discussed at the meeting included the recording of data, and recommendations are made in the report on the essential information required if accounts of chromosome abnormalities and variation are to have maximum value. The place of autoradiography in chromosome identification was considered as well as problems related to the automation of chromosome analysis, population cytogenetics-and in particular the types of population suitable for study and the information which might be derived from such studiesand the effects of radiation and mutagenic substances on chromosomes.

The conference needs following up in two important respects. Its recommendation "that in view of the expansion in human cytogenetics during the past six years it is now desirable to explore the feasibility of establishing a central organization for collecting and analysing data on the variability of the human karyotype" should be acted on by the appropriate international and national bodies. Secondly, its report is available. If investigations and journals publishing papers in this and related fields would use the system of notation recommended in the report then the clarity of published work would be considerably improved. In the past there has been confusion, and valuable data have been lost by the publication of inadequate and incomplete case reports. Widespread acceptance of the recommendations of the Chicago conference should make this a thing of the past.

\section{Health Priorities}

The Ministry of Health's team visiting the U.S.A. and Canada to persuade British medical emigrants to return is now at work. Can it offer the attraction that conditions are so different from what they were, or what the emigrants thought they were, that some will be tempted to return? The Ministry's views, as expressed by its Chief Medical Officer, Sir George Godber, in his annual report, ${ }^{1}$ are robustly hopeful of better times ahead for doctors working in both hospital and general practice. That the department has its views also on what the profession should be doing is apparent from Sir George's observation that, "Given an acceptable and workable opportunity ... the medical profession has shown its ability to work constructively and successfully with the department."

In his introduction Sir George dwells on the year he is reviewing, 1966, as one that " may well be seen in retrospect

1 On the State of the Public Health, The Annual Report of the Chief Medical Officer of the Ministry of Health for the Year 1966. 1967. H.M.S.O.

${ }^{2}$ Management Functions of Hospital Doctors, Ministry of Health, 1966. H.M.S.O. 